\title{
Lorenz comparisons of nine rules for the adjudication of conflicting claims
}

\author{
Kristof Bosmans • Luc Lauwers
}

Accepted: 2 December 2010 / Published online: 5 January 2011

(C) The Author(s) 2011. This article is published with open access at Springerlink.com

\begin{abstract}
Consider the following nine rules for adjudicating conflicting claims: the proportional, constrained equal awards, constrained equal losses, Talmud, Piniles', constrained egalitarian, adjusted proportional, random arrival, and minimal overlap rules. For each pair of rules in this list, we examine whether or not the two rules are Lorenz comparable. We allow the comparison to depend upon whether the amount to divide is larger or smaller than the half-sum of claims. In addition, we provide Lorenz-based characterizations of the constrained equal awards, constrained equal losses, Talmud, Piniles', constrained egalitarian, and minimal overlap rules.
\end{abstract}

Keywords Claims problem - Bankruptcy · Taxation · Lorenz dominance · Proportional rule $\cdot$ Constrained equal awards rule $\cdot$ Constrained equal losses rule Talmud rule · Piniles' rule · Constrained egalitarian rule · Adjusted proportional rule · Random arrival rule $\cdot$ Minimal overlap rule

JEL Classification D63

An earlier version of this paper was circulated in January 2007 (CES Discussion Paper 07.05, Katholieke Universiteit Leuven).

K. Bosmans $(\varangle)$

Department of Economics, Maastricht University, Tongersestraat 53,

6211 LM Maastricht, The Netherlands

e-mail: k.bosmans@maastrichtuniversity.nl

K. Bosmans · L. Lauwers

Center for Economic Studies, Katholieke Universiteit Leuven, Naamsestraat 69,

3000 Leuven, Belgium

e-mail: luc.lauwers@econ.kuleuven.be 


\section{Introduction}

How should an amount of money be divided among a group of individuals if the amount available falls short of the sum of the individuals' claims? Several distribution problems take the form of this "claims problem." Two typical examples are the problems of bankruptcy and taxation. In the case of bankruptcy, the amount to divide is the liquidation value of the firm that goes bankrupt, and the claims are the entitlements of the creditors. In the case of taxation, the amount to divide is the difference between the total pre-tax income and the tax revenue, and the claims are the pre-tax incomes. The literature on the claims problem is largely devoted to the axiomatic study of rules, which associate with each possible claims problem a division among the individuals. ${ }^{1}$

Different rules propose different divisions. We try to detect patterns behind these differences. In particular, we use the Lorenz dominance criterion to check whether a rule is more favourable to smaller claimants relative to larger claimants. ${ }^{2}$ As an illustration, let us consider a claims problem involving three individuals with claims equal to 500, 2000, and 3500, and an amount to divide equal to 1500. Table 1 presents the divisions proposed for this claims problem by the nine rules that we consider: the proportional $(P)$, constrained equal awards $(C E A)$, constrained equal losses $(C E L)$, Talmud $(T)$, Piniles' ( $P$ in $)$, constrained egalitarian $(C E)$, adjusted proportional $(A)$, random arrival $(R A)$, and minimal overlap $(M O)$ rules. The final row of the table presents totals, with the total claim equal to 6000 and the amount to divide equal to 1500 .

As we move from left to right in Table 1, the award allocated to the smallest claimant decreases, while the award allocated to the largest claimant increases. Hence, for each pair of divisions in the table, the left division is obtained from the right division by transferring money from larger claimants to smaller claimants. Equivalently, the left division Lorenz dominates the right division. So for this specific claims problem we may conclude, say, that the Talmud division is more favourable to smaller claimants than the proportional division. The key question is whether this type of conclusion holds in general or depends on the particular data.

The next theorem-which is proven in Sect. 3-summarizes a first set of results. A rule $R$ is said to Lorenz dominate a rule $R^{\prime}$ if, for each claims problem, the division proposed by $R$ Lorenz dominates the division proposed by $R^{\prime}$.

Theorem 1 The Lorenz dominance relation ranks the nine rules as follows:

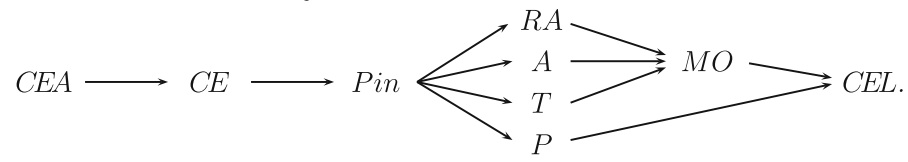

An arrow (or a sequence of arrows) from $R$ to $R^{\prime}$ indicates that $R$ Lorenz dominates $R^{\prime}$. The absence of an arrow (or of a sequence of arrows) indicates the absence of a Lorenz relationship.

\footnotetext{
1 See Moulin (2002) and Thomson (2003) for surveys.

2 The Lorenz criterion is a key concept in the literature on income distribution. See, e.g., Sen (1973).
} 
Table 1 Example with an amount to divide of 1500

\begin{tabular}{rrrrrrr}
\hline Claims & $C E A$ & $C E$, Pin, $T$ & $A$ & $R A, M O$ & $P$ & $C E L$ \\
\hline 500 & 500 & 250 & 214 & 167 & 125 & 0 \\
2000 & 500 & 625 & 643 & 667 & 500 & 0 \\
3500 & 500 & 625 & 643 & 667 & 875 & 1500 \\
\hline 6000 & 1500 & 1500 & 1500 & 1500 & 1500 & 1500 \\
\hline
\end{tabular}

Table 2 Example with an amount to divide of 4500

\begin{tabular}{rlrrrrrrr}
\hline Claims & CEA, CE & Pin & \multicolumn{1}{c}{$P$} & \multicolumn{1}{c}{ RA } & \multicolumn{1}{c}{$A$} & \multicolumn{1}{c}{$T$} & MO & $C E L$ \\
\hline 500 & 500 & 500 & 375 & 333 & 286 & 250 & 167 & 0 \\
2000 & 2000 & 1625 & 1500 & 1333 & 1357 & 1375 & 1417 & 1500 \\
3500 & 2000 & 2375 & 2625 & 2833 & 2857 & 2875 & 2917 & 3000 \\
\hline 6000 & 4500 & 4500 & 4500 & 4500 & 4500 & 4500 & 4500 & 4500 \\
\hline
\end{tabular}

A comment on the interpretation of this result is in order. The fact that a given rule Lorenz dominates another rule should not be regarded as implying that the rule is superior or inferior to the other rule. How to treat larger claimants relative to smaller claimants is a question open to ethical disagreement. Moreover, even the same observer is likely to prefer a different treatment depending on the specific real-world context of the claims problem, e.g., depending on whether it is a case of bankruptcy, taxation, estate division, and so on. ${ }^{3}$

Theorem 1 shows that some rules are Lorenz incomparable. This is the case, for instance, for the proportional rule and the Talmud rule. To illustrate this point, we consider a claims problem with claims as in Table 1, but with an amount to divide of 4500 instead of 1500 . The divisions proposed by the different rules are given in Table 2.

While in Table 1 the Talmud division Lorenz dominates the proportional division, the converse is true in Table 2. As some rules_-viz., the Talmud, Piniles', and constrained egalitarian rules-explicitly treat claims problems differently according to whether the amount to divide is smaller or larger than the half-sum of claims, it seems natural to consider restrictions of the Lorenz dominance relation on these two subsets of claims problems. Considerably more rules are Lorenz comparable on these restricted domains. For instance, for each claims problem with an amount to divide smaller than the half-sum of claims, the Talmud division Lorenz dominates the proportional division, whereas the converse relation holds if the amount available is larger than the half-sum of claims. The results for the restricted domains are summarized in Theorem 2, which is stated and proven in Sect. 4.

\footnotetext{
3 Questionnaire studies confirm that respondents propose different divisions for formally identical claims problems (i.e., involving the same vector of claims and amount to divide) if they are framed in different real-world contexts. For instance, Bosmans and Schokkaert (2009) report that respondents choose divisions more favourable to smaller claimants in a context of pensions than in a context of savings in a firm.
} 
In addition to providing the Lorenz relationships between the nine rules, we characterize six of them as maximal or minimal with respect to the Lorenz dominance relation, i.e., as most or least favourable to smaller claimants: the constrained equal awards, constrained equal losses, Talmud, Piniles', constrained egalitarian, and minimal overlap rules. For instance, we show that the minimal overlap rule is Lorenz-minimal in the set of rules satisfying order preservation of awards, order preservation of losses, reasonable lower bounds on awards, limited consistency, and order preservation under claims variations. The propositions in Sects. 3 and 4 formulate these characterization results and Table 4 provides a summary.

Related results have been established in the literature. ${ }^{4}$ Hougaard and ThorlundPetersen (2001) provide Lorenz rankings of the proportional, constrained equal awards, constrained equal losses, and Talmud rules. Thomson $(2002,2011)$ further discusses the interpretation of Lorenz-based results and ranks the members of the family of increasing-constant-increasing rules, which includes the constrained equal awards, constrained equal losses, Talmud, and minimal overlap rules. Moreno-Ternero and Villar (2006) rank the family of TAL-rules-a subclass of the increasing-constantincreasing family - which includes the constrained equal awards, constrained equal losses, and Talmud rules. Our Lorenz-based characterizations of the constrained equal awards, constrained egalitarian, and Talmud rules are closely related to results by Schummer and Thomson (1997), Chun et al. (2001), and Hougaard and ThorlundPetersen (2001), respectively.

\section{Nine rules and ten properties ${ }^{5}$}

An amount $E$ in $\mathbb{R}_{+}$has to be divided among a set $N=\{1,2, \ldots, n\}$ of at least two individuals with claims adding up to more than $E$. Let $c_{i}$ in $\mathbb{R}_{+}$be individual $i$ 's claim and let $c=\left(c_{1}, c_{2}, \ldots, c_{n}\right)$ be the claims vector. Claims are ordered so that $0 \leq c_{1} \leq c_{2} \leq \cdots \leq c_{n}$. The total claim $c_{1}+c_{2}+\cdots+c_{n}$ is assumed to be positive and is denoted by $C$. A claims problem is an ordered pair $(c, E)$ with $C \geq E$. The set $\mathcal{C}$ collects all claims problems involving $n$ individuals. A rule is a map from the set $\mathcal{C}$ to the set $\mathbb{R}_{+}^{n}$, i.e.,

$$
R: \mathcal{C} \longrightarrow \mathbb{R}_{+}^{n}:(c, E) \longmapsto R(c, E),
$$

that satisfies the conditions $R_{1}(c, E)+R_{2}(c, E)+\cdots+R_{n}(c, E)=E$ and $0 \leq$ $R_{i}(c, E) \leq c_{i}$ for each $i$ in $N$. We refer to $R_{i}(c, E)$ as individual $i$ 's award and to $R(c, E)$ as the awards vector. Sometimes we use $R_{i}$ as shorthand for $R_{i}(c, E)$. We refer to the difference $c_{i}-R_{i}(c, E)$ as individual $i$ 's loss.

\footnotetext{
4 Thomson (2011) provides further references on the use of the Lorenz criterion in this literature.

5 For more details on the rules and properties defined in this section, see Thomson's (2003) survey.
} 


\subsection{Nine rules}

We list nine rules, starting with the four classical ones. ${ }^{6}$ The most commonly used rule in practice makes awards proportional to claims.

Proportional rule, $\boldsymbol{P}$. For each $(c, E)$ in $\mathcal{C}$, we have $P(c, E)=\frac{E}{C} c$.

The next two rules both implement the idea of equality, albeit in different ways. The constrained equal awards rule equalizes awards under the constraint that no individual's award exceeds her claim.

Constrained equal awards rule, $\boldsymbol{C E A}$. For each $(c, E)$ in $\mathcal{C}$ and each $i$ in $N$, we have $C E A_{i}(c, E)=\min \left\{c_{i}, \lambda\right\}$, where $\lambda$ is chosen so that $\sum_{i=1}^{n} \min \left\{c_{i}, \lambda\right\}=E$.

The constrained equal losses rule equalizes losses under the constraint that no award is negative.

Constrained equal losses rule, $\boldsymbol{C E L}$. For each $(c, E)$ in $\mathcal{C}$ and each $i$ in $N$, we have $C E L_{i}(c, E)=\max \left\{0, c_{i}-\lambda\right\}$, where $\lambda$ is chosen so that $\sum_{i=1}^{n} \max \left\{0, c_{i}-\lambda\right\}=E$.

The fourth classical rule, known as the Talmud rule, specifies two regimes depending upon whether or not the amount to divide exceeds the half-sum of the claims. If the amount available is less than the half-sum, then the Talmud rule coincides with the constrained equal awards rule applied to the vector of half-claims. If the amount available is larger than the half-sum, then the Talmud rule gives each individual her half-claim and divides the remainder by applying the constrained equal losses rule to the vector of half-claims.

Talmud rule, $\boldsymbol{T}$. For each $(c, E)$ in $\mathcal{C}$, we have that

(i) if $C / 2 \geq E$, then $T(c, E)=C E A\left(\frac{1}{2} c, E\right)$,

(ii) if $C / 2 \leq E$, then $T(c, E)=\frac{1}{2} c+C E L\left(\frac{1}{2} c, E-C / 2\right)$.

Typical awards vector for the Talmud rule are $\left(\frac{1}{2} c_{1}, \frac{1}{2} c_{2}, \ldots, \frac{1}{2} c_{k}, \lambda, \lambda, \ldots, \lambda\right)$ if $C / 2 \geq E$, and $\left(\frac{1}{2} c_{1}, \frac{1}{2} c_{2}, \ldots, \frac{1}{2} c_{k}, c_{k+1}-\lambda, c_{k+2}-\lambda, \ldots, c_{n}-\lambda\right)$ if $C / 2 \leq E$. If the amount to divide is equal to the half-sum of claims, then the awards vector coincides with the vector of half-claims.

We continue with two rules that coincide with the Talmud rule whenever the amount available is less than the half-sum of the claims. To illuminate the difference with the Talmud rule, we present a typical awards vector for the case where the amount available exceeds the half-sum of claims.

Piniles' rule, Pin. For each $(c, E)$ in $\mathcal{C}$, we have that

(i) if $C / 2 \geq E$, then $\operatorname{Pin}(c, E)=T(c, E)=C E A\left(\frac{1}{2} c, E\right)$,

(ii) if $C / 2 \leq E$, then $\operatorname{Pin}(c, E)=\frac{1}{2} c+C E A\left(\frac{1}{2} c, E-C / 2\right)$.

If $C / 2<E$, then $\left(c_{1}, c_{2}, \ldots, c_{k}, \frac{1}{2} c_{k+1}+\lambda, \frac{1}{2} c_{k+2}+\lambda, \ldots, \frac{1}{2} c_{n}+\lambda\right)$ is a typical awards vector for Piniles' rule.

\footnotetext{
6 Herrero and Villar (2001) provide a comparative examination of these four rules.
} 
Constrained egalitarian rule, $\boldsymbol{C E}$. For each $(c, E)$ in $\mathcal{C}$, we have that

(i) if $C / 2 \geq E$, then $C E(c, E)=T(c, E)=C E A\left(\frac{1}{2} c, E\right)$,

(ii) if $C / 2 \leq E$, then, for each $i$ in $N$, we have $C E_{i}(c, E)=\max \left\{c_{i} / 2, \min \left\{c_{i}, \lambda\right\}\right\}$, where $\lambda$ is chosen so that $\sum_{i=1}^{n} \max \left\{c_{i} / 2, \min \left\{c_{i}, \lambda\right\}\right\}=E$.

If $C / 2<E$, then $\left(c_{1}, c_{2}, \ldots, c_{k}, \lambda, \lambda, \ldots, \lambda, \frac{1}{2} c_{\ell}, \frac{1}{2} c_{\ell+1}, \ldots, \frac{1}{2} c_{n}\right)$

is a typical awards vector for the constrained egalitarian rule.

The final three rules coincide with the Talmud rule in the case of two individuals. The adjusted proportional rule first allocates to each individual her minimal right, i.e., the part of the amount to divide that is left after each other individuals is fully compensated (such a minimal right might be zero). Next, the claims and the amount to divide are revised (with the revised claims furthermore truncated by the revised amount to divide) and the resulting problem is solved using the proportional rule. Formally, for the claims problem $(c, E)$, individual $i$ 's minimal right $m_{i}(c, E)$ is defined as $\max \left\{0, E-C+c_{i}\right\}$ and $m(c, E)=\left(m_{1}(c, E), m_{2}(c, E), \ldots, m_{n}(c, E)\right)$. The adjusted amount to divide $E-\sum_{i=1}^{n} m_{i}(c, E)$ is denoted by $E_{A}$.

Adjusted proportional rule, $\boldsymbol{A}$. For each $(c, E)$ in $\mathcal{C}$, we have

$$
A(c, E)=m(c, E)+P\left(\left(\min \left\{c_{i}-m_{i}(c, E), E_{A}\right\}\right)_{i \in N}, E_{A}\right) .
$$

To define the next rule, assume the individuals arrive one by one, each receiving full compensation until the money runs out. By averaging the awards vectors obtained in this way over all possible orders of arrival, we get the division proposed by the random arrival rule. Let $\mathcal{P}$ collect the $n$ ! different orderings in the set $N$. For each ordering $\pi$ in $\mathcal{P}$ and for each individual $i$ in $N$, the set $\pi[i]$ collects the predecessors of $i$ with respect to the ordering $\pi$. $^{7}$

Random arrival rule, $\boldsymbol{R} \boldsymbol{A}$. For each $(c, E)$ in $\mathcal{C}$ and for each $i$ in $N$, we have

$$
R A_{i}(c, E)=\frac{1}{n !} \sum_{\pi \in \mathcal{P}} \min \left\{c_{i}, \max \left\{0, E-\sum_{j \in \pi[i]} c_{j}\right\}\right\} .
$$

For the definition of the minimal overlap rule, we follow Chun and Thomson (2005, p. 138). The rule distinguishes two cases. In case (i), there exists an individual with a claim larger than or equal to the amount to divide $E$. Each individual is regarded as claiming an interval rather than an abstract amount. In particular, an individual $i$ with a claim smaller than $E$ claims the interval $\left[0, c_{i}\right]$, and an individual with a claim larger than or equal to $E$ claims the interval $[0, E]$. Next, each part of the interval $[0, E]$ is divided equally among all individuals claiming it. For instance, the interval $\left[0, c_{1}\right]$ is claimed by all individuals, and so each receives $c_{1} / n$. The interval $\left(c_{1}, c_{2}\right]$ is claimed by all individuals except individual 1 , and so each member of $N-\{1\}$ receives in addition $\left(c_{2}-c_{1}\right) /(n-1)$. This process continues until the entire interval $[0, E]$ is distributed. In case (ii), all individuals have claims smaller than the amount to divide.

\footnotetext{
7 For example, let $N=\{1,2,3\}$ and consider the ordering $\pi=(1,3,2)$ in which individual 1 queues first, followed by 3 , and finally 2 . In this case, $\pi[1]=\varnothing, \pi[2]=\{1,3\}$, and $\pi[3]=\{1\}$.
} 
We let $c_{0}=0$ and look for the largest $k^{*}$ in $\{0,1,2, \ldots, n-2\}$ for which there exists a $t$ in $\mathbb{R}_{+}$that satisfies

$$
c_{k^{*}}<t \leq c_{k^{*}+1} \text { and }\left(c_{k^{*}+1}-t\right)+\left(c_{k^{*}+2}-t\right)+\cdots+\left(c_{n}-t\right)=E-t .8
$$

Each individual $i$ in the set $\left\{k^{*}+1, k^{*}+2, \ldots, n\right\}$ receives a first share equal to $c_{i}-t$, i.e., the part of the interval $(t, E]$ that $i$ alone claims. The remaining part $[0, t]$ is divided as in case (i) with $t$ as the amount to divide.

Minimal overlap rule, $\boldsymbol{M O}$. Let $c_{0}=0$. For each $(c, E)$ in $\mathcal{C}$, we have the following.

(i) Let $c_{k^{*}}<E \leq c_{k^{*}+1} \leq c_{n}$ with $k^{*}$ in $\{0,1,2, \ldots, n-1\}$. Then,

$$
\begin{aligned}
M O_{i} & =\frac{c_{1}}{n}+\frac{c_{2}-c_{1}}{n-1}+\frac{c_{3}-c_{2}}{n-2}+\cdots+\frac{c_{i}-c_{i-1}}{n-i+1} \text { for each } i=1,2, \ldots, k^{*}, \\
M O_{j} & =M O_{k^{*}}+\frac{E-c_{k^{*}}}{n-k^{*}} \text { for each } j=k^{*}+1, k^{*}+2, \ldots, n
\end{aligned}
$$

(ii) Let $c_{n}<E$. Let $c_{k^{*}}<t \leq c_{k^{*}+1}$ with $k^{*}$ in $\{0,1,2, \ldots, n-2\}$ and $t$ as in (1). Then,

$$
\begin{aligned}
& M O_{i}=\frac{c_{1}}{n}+\frac{c_{2}-c_{1}}{n-1}+\frac{c_{3}-c_{2}}{n-2}+\cdots+\frac{c_{i}-c_{i-1}}{n-i+1} \text { for each } i=1,2, \ldots, k^{*}, \\
& M O_{j}=\left(c_{j}-t\right)+M O_{k^{*}}+\frac{t-c_{k^{*}}}{n-k^{*}} \text { for each } j=k^{*}+1, k^{*}+2, \ldots, n .
\end{aligned}
$$

\subsection{Ten properties}

We consider ten properties. Table 3 indicates which of these properties are satisfied by each of the nine rules defined in the previous subsection.

Order preservation of awards requires that awards are ordered as claims are.

Order preservation of awards. For each $(c, E)$ in $\mathcal{C}$, we have that if $c_{i} \leq c_{j}$, then $R_{i}(c, E) \leq R_{j}(c, E)$.

Order preservation of losses demands that losses are ordered as claims are.

Order preservation of losses. For each $(c, E)$ in $\mathcal{C}$, we have that if $c_{i} \leq c_{j}$, then $c_{i}-R_{i}(c, E) \leq c_{j}-R_{j}(c, E)$.

Resource monotonicity holds that if the amount to divide increases, then each individual should receive at least as much as she did initially.

Resource monotonicity. For each pair $(c, E)$ and $\left(c, E^{\prime}\right)$ in $\mathcal{C}$, we have that if $E \leq$ $E^{\prime} \leq C$, then $R(c, E) \leq R\left(c, E^{\prime}\right)$.

The next two properties describe responses of the awards vector to changes in the amount available and in the claims vector, respectively. Super-modularity requires that if the amount to divide increases, given two individuals, the one with the greater claim

\footnotetext{
${ }^{8}$ If the claims happen to be feasible, i.e., $c_{1}+c_{2}+\cdots+c_{n}=E$, then $k^{*}=0$ and we allow $t=c_{0}=0$.
} 
Table 3 Rules and properties (for at least three individuals)

\begin{tabular}{lccccccccc}
\hline & $P$ & $C E A$ & $C E L$ & $T$ & Pin & $C E$ & $A$ & $R A$ & $M O$ \\
\hline Order preservation of awards & Yes & Yes & Yes & Yes & Yes & Yes & Yes & Yes & Yes \\
Order preservation of losses & Yes & Yes & Yes & Yes & Yes & Yes & Yes & Yes & Yes \\
Resource monotonicity & Yes & Yes & Yes & Yes & Yes & Yes & Yes & Yes & Yes \\
Super-modularity & Yes & Yes & Yes & Yes & Yes & No & Yes & Yes & Yes \\
Order preservation under claims vari- & Yes & Yes & Yes & Yes & Yes & No & Yes & Yes & Yes \\
ations & & & & & & & & & \\
Invariance under claims truncation & No & Yes & No & Yes & Yes & Yes & Yes & Yes & Yes \\
Self-duality & Yes & No & No & Yes & No & No & Yes & Yes & No \\
Midpoint property & Yes & No & No & Yes & Yes & Yes & Yes & Yes & No \\
Limited consistency & Yes & Yes & Yes & Yes & Yes & Yes & Yes & Yes & Yes \\
Reasonable lower bounds on awards & No & Yes & No & Yes & Yes & Yes & Yes & Yes & Yes \\
\hline
\end{tabular}

experiences a larger gain than the other. Order preservation under claims variations (Thomson 2006, p. 106) requires that if the claim of some individual decreases, given two other individuals, the one with the greater claim experiences a larger gain than the other.

Super-modularity. For each pair $(c, E)$ and $\left(c, E^{\prime}\right)$ in $\mathcal{C}$ with $E \leq E^{\prime} \leq C$, and for each pair $i$ and $j$ in $N$ with $c_{i} \leq c_{j}$, we have $R_{i}\left(c, E^{\prime}\right)-R_{i}(c, E) \leq R_{j}\left(c, E^{\prime}\right)-$ $R_{j}(c, E)$.

We write $\left(c_{k}^{\prime}, c_{-k}\right)$ for the claims vector obtained from $c$ by replacing $c_{k}$ with $c_{k}^{\prime}$.

Order preservation under claims variations. For each $k$ in $N$, for each pair $(c, E)$ and $\left(c^{\prime}, E\right)$ in $\mathcal{C}$ with $c^{\prime}=\left(c_{k}^{\prime}, c_{-k}\right)$ and $c_{k}^{\prime}<c_{k}$, and for each pair $i$ and $j$ in $N-\{k\}$ with $c_{i} \leq c_{j}$, we have $R_{i}\left(c^{\prime}, E\right)-R_{i}(c, E) \leq R_{j}\left(c^{\prime}, E\right)-R_{j}(c, E)$.

We postpone the discussion of which rules satisfy order preservation under claims variations to the end of this subsection.

The next property requires that truncating the claims at the level of the amount to divide has no impact on awards.

Invariance under claims truncation. For each claims problem $(c, E)$ in $\mathcal{C}$, we have $R(c, E)=R\left(\left(\min \left\{c_{i}, E\right\}\right)_{i \in N}, E\right)$.

A self-dual rule treats the problem of dividing the amount available and the problem of dividing the shortfall (i.e., the difference between the total claim and the amount to divide) in a symmetrical way.

Self-duality. For each $(c, E)$ in $\mathcal{C}$, we have $R(c, E)=c-R(c, C-E)$.

The adjusted proportional rule inherits self-duality from the proportional rule (Thomson and Yeh 2006, Corollary 1).

The midpoint property requires the awards vector to coincide with the vector of half-claims whenever the amount to divide coincides with the half-sum of claims.

Midpoint property. For each $(c, E)$ in $\mathcal{C}$ with $E=C / 2$, we have $R(c, E)=\frac{1}{2} c$.

Self-duality implies the midpoint property. Indeed, if $R(c, E)+R(c, C-E)=c$, then, for a claims problem $(c, E)$ with $C=2 E$, we have $2 R(c, E)=c$. 
Limited consistency states that adding an individual with a zero claim does not change the awards of the individuals already present. Abusing notation, we let $R$ denote both the $n$-person and the $(n+1)$-person version of a rule. Obviously, if $\left(c_{1}, c_{2}, \ldots, c_{n}, E\right)$ is a claims problem involving $n$ individuals, then $\left(0, c_{1}, c_{2}, \ldots, c_{n}, E\right)$ is a claims problem with $n+1$ individuals.

Limited consistency. For each $(c, E)=\left(c_{1}, c_{2}, \ldots, c_{n}, E\right)$ in $\mathcal{C}$ involving $n$ individuals, we have $R\left(0, c_{1}, c_{2}, \ldots, c_{n}, E\right)=\left(0, R\left(c_{1}, c_{2}, \ldots, c_{n}, E\right)\right)$.

Finally, reasonable lower bounds on awards (Moreno-Ternero and Villar 2004; Dominguez and Thomson 2006) ensures that each individual receives at least the minimum of (i) her claim divided by the number of individuals and (ii) the amount available divided by the number of individuals.

Reasonable lower bounds on awards. For each $(c, E)$ in $\mathcal{C}$ and for each $i$ in $N$, we have $R_{i}(c, E) \geq \min \left\{c_{i}, E\right\} / n^{*}$, where $n^{*}$ denotes the number of individuals with a positive claim.

We now turn to the question of which rules satisfy order preservation under claims variations. First, it can easily be established that each consistent ${ }^{9}$ rule satisfies supermodularity if and only if it satisfies order preservation under claims variations. The proportional, constrained equal awards, constrained equal losses, Talmud, and Piniles' rules all satisfy consistency and super-modularity, and therefore satisfy order preservation under claims variations. The constrained egalitarian rule, on the other hand, is consistent but does not satisfy super-modularity. Hence, it does not satisfy order preservation under claims variations.

We now consider the adjusted proportional rule. The larger the claim is, the larger the minimal right. If one of the claims decreases, then the minimal rights of the other individuals increase in an order preserving manner (the minimal right either stays at the zero level, or becomes positive, or increases with the amount the particular claim decreases). The proportional rule is applied to the adjusted claims problem, and its proposal is added to the minimal rights vector. Since, moreover, the proportional rule satisfies order preservation under claims variations, the adjusted proportional rule also satisfies the property.

Next, we check whether the random arrival rule satisfies order preservation under claims variations. Let $(c, E)$ be a claims problem, let $c_{k}$ decrease to $c_{k}^{\prime}$, and let $i$ and $j$ be two individuals different from $k$ such that $c_{i} \leq c_{j}$. Consider an order of arrival $\pi$ in which the decrease in $c_{k}$ generates an increase in individual $i$ 's award (i.e., $k \in \pi[i]$ and $0<E-\sum_{\ell \in \pi[i]} c_{\ell}<c_{i}$ ). Switch the positions of $i$ and $j$ in $\pi$ and obtain the order $\pi^{\prime}$. Then, $k \in \pi^{\prime}[j]$ and $0<E-\sum_{\ell \in \pi^{\prime}[j]} c_{\ell}<c_{j}$. With respect to the order $\pi^{\prime}$, individual $j$ experiences at least the same increase as $i$ does with respect to $\pi$. By consequence, the random arrival rule satisfies order preservation under claims variations.

Finally, the minimal overlap rule also satisfies order preservation under claims variations, as will become clear in the proof of Proposition 4.

\footnotetext{
9 See Thomson (2003, p. 279) for a definition of the consistency property.
} 


\section{Lorenz comparisons on the full domain}

In this section we prove Theorem 1. First, we define the Lorenz dominance relation. Let $\mathbb{R}_{\leq}^{n}$ be the set of nonnegative $n$-dimensional vectors $x=\left(x_{1}, x_{2}, \ldots, x_{n}\right)$ ordered from small to large, i.e., $0 \leq x_{1} \leq x_{2} \leq \cdots \leq x_{n}$. Let $x$ and $y$ be in $\mathbb{R}_{\leq}^{n}$. We say that $x$ Lorenz dominates $y$ if we have

$$
x_{1}+x_{2}+\cdots+x_{k} \geq y_{1}+y_{2}+\cdots+y_{k} \text { for each } k=1,2, \ldots, n-1,
$$

and $x_{1}+x_{2}+\cdots+x_{n}=y_{1}+y_{2}+\cdots+y_{n}$. If $x$ Lorenz dominates $y$ and $x \neq y$, then at least one of these $n-1$ inequalities is a strict inequality. The following definition extends the notion of Lorenz dominance to the domain of rules.

Definition Let $R$ and $R^{\prime}$ be two rules that satisfy order preservation of awards and let $\mathcal{D} \subseteq \mathcal{C}$ be a set of claims problems. Then, $R$ Lorenz dominates $R^{\prime}$ on the domain $\mathcal{D}$ if $R(c, E)$ Lorenz dominates $R^{\prime}(c, E)$ for each $(c, E)$ in $\mathcal{D}$.

Since Lorenz dominance is applied to awards vectors, the restriction to rules satisfying order preservation of awards is required to allow the interpretation of Lorenz dominance in terms of favourability to smaller claimants. We shorten "Lorenz dominance on the domain $\mathcal{C}$ " to "Lorenz dominance." The transitivity and reflexivity of the Lorenz dominance relation in the set $\mathbb{R}_{\leq}^{n}$ implies the transitivity and reflexivity of the Lorenz dominance relation in the set of rules.

According to the next lemma, the duality operator reverses the Lorenz dominance relation. The dual rule $R^{d}$ of $R$ treats what is available for division in the same way as $R$ treats what is missing. Formally, for each $(c, E)$ in $\mathcal{C}$, we have $R^{d}(c, E)=$ $c-R(c, C-E)$. The claims problems $(c, C-E)$ and $(c, E)$ are said to be dual.

Lemma 1 Let $R$ and $S$ be two rules that satisfy order preservation of awards and order preservation of losses, and let $\mathcal{D} \subseteq \mathcal{C}$ be a set of claims problems. Then, $R$ Lorenz dominates $S$ on the domain $\mathcal{D}$ if and only if $S^{d}$ Lorenz dominates $R^{d}$ on the domain $\mathcal{D}^{d}$ of dual claims problems.

Proof Let $(c, E)$ be a claims problem in $\mathcal{D}$. Then, $(c, C-E)$ belongs to $\mathcal{D}^{d}$. Duality implies

$$
R(c, E)+R^{d}(c, C-E)=c \text { and } S(c, E)+S^{d}(c, C-E)=c .
$$

Conclude that $R(c, E)$ Lorenz dominates $S(c, E)$ if and only if $S^{d}(c, C-E)$ Lorenz dominates $R^{d}(c, C-E)$.

The rest of this section proves the Lorenz relationships indicated by the arrows in Theorem 1 (see Sect. 1). We proceed from left to right and from top to bottom. The examples in Tables 1 and 2 demonstrate the incomparabilities. A Lorenz-based characterization of a rule is stated as a proposition.

(a) $C E A \rightarrow C E$. This Lorenz comparison follows from the fact that the constrained equal awards rule is Lorenz-maximal in the set of rules that preserve the order of awards. 
Proposition 1 Let $\mathcal{R}$ be the set of rules that satisfy order preservation of awards. The constrained equal awards rule is the only rule in $\mathcal{R}$ that Lorenz dominates each rule in $\mathcal{R} .^{10}$

Proof It suffices to show that $C E A$ Lorenz dominates each rule in $\mathcal{R}$. This is done by contradiction. Let $R$ in $\mathcal{R}$ and $(c, E)$ in $\mathcal{C}$ be such that $x=C E A(c, E)$ does not Lorenz dominate $y=R(c, E)$. Let $k$ in $N$ be the smallest number such that $x_{1}+x_{2}+\cdots+x_{k}<y_{1}+y_{2}+\cdots+y_{k}$.

Hence, $x_{k}<y_{k} \leq c_{k}$. Therefore, $x_{k}=\lambda$ (according to CEA the individuals that receive less than $\lambda$ are fully compensated). As $\lambda<y_{k}$ and $y_{k} \leq y_{k+1} \leq \cdots \leq y_{n}$ (the rule $R$ preserves the order of awards), the allocation $y$ is not feasible.

(b) $C E \rightarrow$ Pin. Recall that the constrained egalitarian and Piniles' rules satisfy resource monotonicity and the midpoint property. The Lorenz comparison of these two rules is established by Chun et al. (2001, Theorem 3). We recall their result and provide a different proof.

Proposition 2 Let $\mathcal{R}$ be the set of rules that satisfy order preservation of awards, the midpoint property, and resource monotonicity. The constrained egalitarian rule is the only rule in $\mathcal{R}$ that Lorenz dominates each rule in $\mathcal{R}$.

Proof It suffices to show that $C E$ Lorenz dominates each rule in $\mathcal{R}$. This is done by contradiction. Let $R$ in $\mathcal{R}$ and $(c, E)$ in $\mathcal{C}$ be such that $x=C E(c, E)$ does not Lorenz dominate $y=R(c, E)$. Let $k$ in $N$ be the smallest number such that $x_{1}+x_{2}+\cdots+x_{k}<$ $y_{1}+y_{2}+\cdots+y_{k}$.

Hence, $x_{k}<y_{k}$ and $x_{\ell}>y_{\ell}$ for some $\ell>k$. The inequalities $x_{k}<y_{k} \leq c_{k}$ imply that either $x_{k}=c_{k} / 2$ or $x_{k}=\lambda<c_{k}$. In addition, $\ell>k$ implies $x_{\ell}<c_{\ell}(C E$ fully compensates only-if any-the smaller claims). We distinguish $C / 2>E$ from $C / 2<E$. The midpoint property tackles the case $C / 2=E$.

Case 1, $C / 2>E$ and $x_{k}=c_{k} / 2$. Then, $y_{k}>x_{k}$ cannot hold because of resource monotonicity and the midpoint property. Indeed, if $E$ increases towards $C / 2$, then $y_{k}$ should increase towards $c_{k} / 2$, and a contradiction follows.

Case 2, $C / 2>E$ and $x_{k}=\lambda$. Then, the inequalities $\lambda<y_{k} \leq y_{k+1} \leq \cdots \leq y_{n}(R$ preserves the order of awards) make the vector $y$ infeasible.

Case 3, $C / 2<E$ and $y_{\ell}<x_{\ell}=c_{\ell} / 2$. Again, a contradiction follows: if $E$ decreases towards $C / 2$, then $y_{\ell}$ should decrease towards $c_{\ell} / 2$.

Case 4, $C / 2<E$ and $y_{\ell}<x_{\ell}=\lambda$. We obtain the configuration $x_{k}=x_{k+1}=\cdots=$ $x_{\ell}=\lambda$. Then, the rule $R$ does not preserve the order of awards: $y_{k}>\lambda$, while $y_{\ell}<\lambda$.

(c) Pin $\rightarrow\{R A, A, T, P\}$. In the case where the half-sum of claims is larger than the amount to divide, Piniles' rule coincides with the constrained egalitarian and Talmud rules. Proposition 2 implies that the constrained egalitarian rule Lorenz dominates the Piniles', random arrival, adjusted proportional, Talmud, and proportional rules. Hence,

10 Proposition 1 entails results by Schummer and Thomson (1997, Propositions 3 and 4). 
on the domain $\mathcal{C}_{1}$ of claims problems $(c, E)$ with $C / 2 \geq E$, the rule $\operatorname{Pin}=T=C E$ Lorenz dominates the random arrival, adjusted proportional, and proportional rules.

We now focus on problems with a half-sum of claims less than the amount to divide. Let $\mathcal{C}_{0}$ collect the problems $(c, E)$ for which $C / 2 \leq E$. Since the Piniles', random arrival, adjusted proportional, Talmud, and proportional rules all satisfy the properties required, the next lemma simultaneously tackles the relationships on the restricted domain $\mathcal{C}_{0}$.

Lemma 2 Restrict the domain to $\mathcal{C}_{0}$. Let $\mathcal{R}$ be the set of rules that satisfy order preservation of awards, the midpoint property, and super-modularity. Piniles' rule ( $\left.\operatorname{Pin}\right|_{\mathcal{C}_{0}}$ ) is the only rule in $\mathcal{R}$ that Lorenz dominates each rule in $\mathcal{R}$ on the domain $\mathcal{C}_{0}$.

Proof It suffices to show that Pin Lorenz dominates each rule in $\mathcal{R}$. This is done by contradiction. Let $R$ in $\mathcal{R}$ and $(c, E)$ in $\mathcal{C}_{0}$ be such that $x=\operatorname{Pin}(c, E)$ does not Lorenz dominate $y=R(c, E)$. Let $k$ in $N$ be the smallest number such that $x_{1}+x_{2}+\cdots+x_{k}<y_{1}+y_{2}+\cdots+y_{k}$.

Hence, $x_{k}<y_{k} \leq c_{k}$ and $y_{\ell}<x_{\ell} \leq c_{\ell}$ for some $\ell>k$. The definition of Pin implies that $x_{k}=c_{k} / 2+\lambda$ and $x_{\ell}=c_{\ell} / 2+\lambda$. As the rule $R$ satisfies the midpoint property, we have $R_{k}(c, E)-R_{k}(c, C / 2)=y_{k}-c_{k} / 2>\lambda$, while $R_{\ell}(c, E)-R_{\ell}(c, C / 2)=$ $y_{\ell}-c_{\ell} / 2<\lambda$. This contradicts the fact that $R$ satisfies super-modularity (recall that $\left.c_{k} \leq c_{\ell}\right)$.

The combination of Lemma 2 and Proposition 2 entails a Lorenz-based characterization of Piniles' rule.

Proposition 3 Let $\mathcal{R}$ be the set of rules that satisfy order preservation of awards, resource monotonicity, the midpoint property, and super-modularity. Piniles' rule is the only rule in $\mathcal{R}$ that Lorenz dominates each rule in $\mathcal{R}$.

(d) $\{R A, A, T\} \rightarrow M O$. If there are only two individuals, then the random arrival, adjusted proportional, Talmud, and minimal overlap rules coincide (with the concedeand-divide rule; see Thomson 2003). The next proposition provides a Lorenz-based characterization of the minimal overlap rule.

Proposition 4 Let $\mathcal{R}$ be the set of rules that satisfy order preservation of awards, order preservation of losses, order preservation under claims variations, limited consistency, and reasonable lower bounds on awards. The minimal overlap rule is the only rule in $\mathcal{R}$ that is Lorenz dominated by each rule in $\mathcal{R}$.

Proof By induction on the number of individuals.

Proof for $n=2$. Let $(c, E)$ be a claims problem with two individuals. If $k^{*}=0$, then either (i) $E \leq c_{1}$ and $M O(c, E)=(E / 2, E / 2)$, or (ii) $M O(c, E)=\left(c_{1}-s, c_{2}-s\right)$ with $s=(C-E) / n$. Since each rule $R$ in $\mathcal{R}$ satisfies (i) reasonable lower bounds and (ii) order preservation of losses, the awards vector $R(c, E)$ Lorenz dominates $M O(c, E)$.

If $k^{*}=1$, i.e., $c_{1}<E \leq c_{2}$, then the minimal overlap rule proposes $x=\left(c_{1} / 2, E-\right.$ $\left.c_{1} / 2\right)$. Each rule that satisfies reasonable lower bounds on awards proposes a division that Lorenz dominates $x$. 
Inductive step. Suppose that the proposition holds for claims problems with at most $n-1$ individuals. We have to show that the proposition holds for claims problems with $n$ individuals. Consider a rule $R$-defined for each number of individuals - in $\mathcal{R}$.

Let $(c, E)$ be a claims problem with $n$ individuals. If $k^{*}=0$, then either (i) $E \leq c_{1}$ and $M O(c, E)=(E / n, E / n, \ldots, E / n)$, or (ii) $M O(c, E)=\left(c_{1}-s, c_{2}-s, \ldots, c_{n}-\right.$ $s)$ with $s=(C-E) / n$. Since each rule $R$ in $\mathcal{R}$ satisfies (i) reasonable lower bounds on awards and (ii) order preservation of losses, the awards vector $R(c, E)$ Lorenz dominates $M O(c, E)$.

If $k^{*}>0$, then $c_{2}+c_{3}+\cdots+c_{n}>E$ and $\left(0, c_{2}, c_{3}, \ldots, c_{n}, E\right)$ is a claims problem. In addition, the $k^{*}$ for problem $\left(0, c_{2}, c_{3}, \ldots, c_{n}, E\right)$ coincides with the original $k^{*}$ for problem $(c, E)$.

The inductive hypothesis implies that the $(n-1)$-dimensional vector $R\left(c_{2}, c_{3}, \ldots, c_{n}, E\right)$ Lorenz dominates $M O\left(c_{2}, c_{3}, \ldots, c_{n}, E\right)$. Since $R$ and $M O$ satisfy limited consistency, we have

$$
\begin{aligned}
& \left(0, R\left(c_{2}, c_{3}, \ldots, c_{n}, E\right)\right) \quad \text { Lorenz dominates }\left(0, M O\left(c_{2}, c_{3}, \ldots, c_{n}, E\right)\right) \text {, } \\
& \text { II } \\
& \text { II } \\
& R\left(0, c_{2}, c_{3}, \ldots, c_{n}, E\right) \quad \text { Lorenz dominates } M O\left(0, c_{2}, c_{3}, \ldots, c_{n}, E\right) \text {. }
\end{aligned}
$$

Start from $\left(0, c_{2}, c_{3}, \ldots, c_{n}, E\right)$ and let the claim of individual 1 increase from 0 to $c_{1}$. The minimal overlap rule transfers an amount $c_{1} /[n(n-1)]$ from each individual $i=2,3, \ldots, n$ to individual 1 , who obtains an award equal to $c_{1} / n$. Because the rule $R$ satisfies reasonable lower bounds on awards, it allocates at least $c_{1} / n$ to individual 1. Furthermore, the rule $R$ satisfies order preservation under claims variations. Hence, when the claim of individual 1 increases from 0 to $c_{1}$, the decrease in the award $R_{i}$ of individual $i \neq 1$ is increasing in $i$. Conclude that $R\left(c_{1}, c_{2}, \ldots, c_{n}, E\right)$ Lorenz dominates $M O\left(c_{1}, c_{2}, \ldots, c_{n}, E\right)$.

(e) $\{M O, P\} \rightarrow C E L$. The constrained equal losses rule is the dual of the constrained equal awards rule (see, e.g., Herrero and Villar 2001). Whereas the constrained equal awards rule is Lorenz-maximal, the constrained equal losses rule is Lorenz-minimal.

Proposition 5 Let $\mathcal{R}$ be the set of rules that satisfy order preservation of awards and order preservation of losses. The constrained equal losses rule is the only rule in $\mathcal{R}$ that is Lorenz dominated by each rule in $\mathcal{R}$.

Proof Note that if a rule satisfies order preservation of awards, then its dual satisfies order preservation of losses. Proposition 1 implies that CEA Lorenz dominates each rule that satisfies order preservation of awards and order preservation of losses. Take the dual of this implication and apply Lemma 1.

This completes the proof of Theorem 1. 


\section{Lorenz comparisons on restricted domains}

Theorem 1 presents an incomplete ranking: the set $\{R A, A, T, P\}$ and the pair $\{P, M O\}$ are not ranked. In this section, we focus on both these sets of rules and study their relationships on the restricted domains $\mathcal{C}_{0.5}(C / 2=E), \mathcal{C}_{0}(C / 2 \leq E)$, and $\mathcal{C}_{1}(C / 2 \geq E)$. As already mentioned, the position of the amount to divide against the half-sum of claims is crucial. We formulate our second theorem.

Theorem 2 On the restricted domains $\mathcal{C}_{0.5}, \mathcal{C}_{0}$, and $\mathcal{C}_{1}$, the Lorenz dominance relation ranks the nine rules as follows:

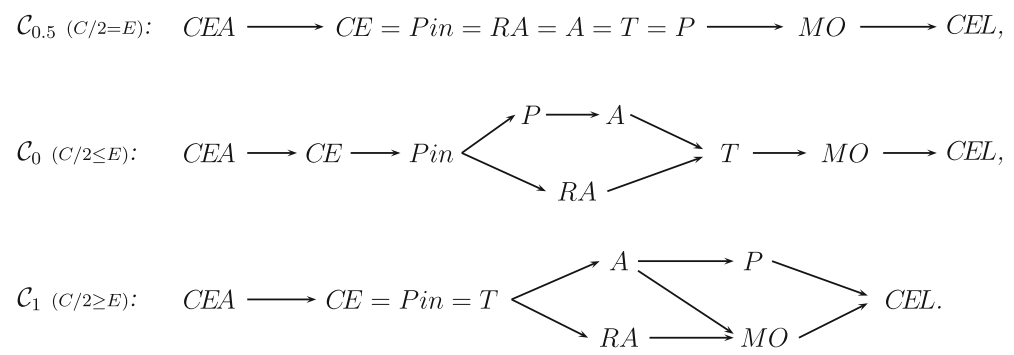

An arrow (or a sequence of arrows) from rule $R$ to $R^{\prime}$ indicates that $R$ Lorenz dominates $R^{\prime}$ on the relevant restricted domain. The absence of an arrow (or of a sequence of arrows) indicates the absence of a Lorenz relationship.

We subsequently discuss the domains $\mathcal{C}_{0.5}, \mathcal{C}_{0}$, and $\mathcal{C}_{1}$, and prove the relationships not covered by Theorem 1 . We distinguish the Lorenz relations by denoting the domain in stack position, e.g., $P \stackrel{\mathcal{C}_{0}}{\longrightarrow} A$.

(a) The domain $\mathcal{C}_{0.5}$. The arrows involving the constrained equal awards, minimal overlap, and constrained equal losses rules are implied by Theorem 1 . The equalities $C E=P i n=R A=A=T=P$ follow from the midpoint property.

We now consider the domains $\mathcal{C}_{0}(C / 2 \leq E)$ and $\mathcal{C}_{1}(C / 2 \geq E)$. Observe that several rankings reverse over these domains: we have $P \stackrel{\mathcal{C}_{0}}{\longrightarrow} A \stackrel{\mathcal{C}_{0}}{\longrightarrow} T$ and $T \stackrel{\mathcal{C}_{1}}{\longrightarrow}$ $A \stackrel{\mathcal{C}_{1}}{\longrightarrow} P$, as well as $R A \stackrel{\mathcal{C}_{0}}{\longrightarrow} T$ and $T \stackrel{\mathcal{C}_{1}}{\longrightarrow} R A .{ }^{11}$ Since the domains $\mathcal{C}_{0}$ and $\mathcal{C}_{1}$ are dual and the proportional, adjusted proportional, Talmud, and random arrival rules are self-dual, these reversals are a consequence of Lemma 1.

(b) $P \stackrel{\mathcal{C}_{0}}{\longrightarrow} A$ and $A \stackrel{\mathcal{C}_{1}}{\longrightarrow} P$. By Lemma 1 and self-duality of the proportional and adjusted proportional rules, we need only consider the second statement. Let $(c, E)$ be a claims problem with $C / 2 \geq E$. If the minimal right $m_{i}$ of individual $i$ is positive, then either $E-C+c_{i}>0$, or $E+c_{i}>C \geq 2 E$, or $c_{i}>E$. By consequence, at most one individual has a positive minimal right. We distinguish two cases.

\footnotetext{
11 The switch from $P \stackrel{\mathcal{C}_{0}}{\longrightarrow} A$ to $A \stackrel{\mathcal{C}_{1}}{\longrightarrow} P$ is especially noteworthy because the proportional and adjusted proportional rules - in contrast to, for instance, the Talmud rule — do not have different recipes for claims problems according to whether the half-sum of claims is larger or smaller than the amount available.
} 
Case $1, m_{n}(c, E)=0$. Then, each individual has a minimal right equal to 0 . The definition of $A$ implies

$$
A(c, E)=A(\bar{c}, E)=P(\bar{c}, E) \text { with } \bar{c}=\left(\min \left\{c_{i}, E\right\}\right)_{i \in N} .
$$

Only the larger claims are truncated. Therefore, the vector $P(\bar{c}, E)$ Lorenz dominates $P(c, E)$.

Case 2, $m_{n}(c, E)>0$. The minimal rights vector reads $m=(0,0, \ldots, 0, E-C+$ $\left.c_{n}\right)$. The adjusted amount to divide $E_{A}$ is equal to $E-m_{n}=C-c_{n}$. Also, $c_{n}-m_{n}>E-m_{n}$. Next, we determine $P\left(c_{1}, c_{2}, \ldots, c_{n-1}, C-c_{n}, C-c_{n}\right)$. As the claims add up to $2\left(C-c_{n}\right)$, we obtain $P\left(c_{1}, c_{2}, \ldots, c_{n-1}, C-c_{n}, C-\right.$ $\left.c_{n}\right)=\left(c_{1} / 2, c_{2} / 2, \ldots, c_{n-1} / 2,\left(C-c_{n}\right) / 2\right)$. By consequence,

$$
A(c, E)=\left(c_{1} / 2, c_{2} / 2, \ldots, c_{n-1} / 2, E-\left(C-c_{n}\right) / 2\right) .
$$

On the other hand, $E / C \leq 1 / 2$ implies $P(c, E) \leq \frac{1}{2} c$. The Lorenz dominance result follows.

(c) $\{A, R A\} \stackrel{\mathcal{C}_{0}}{\longrightarrow} T$ and $T \stackrel{\mathcal{C}_{1}}{\longrightarrow}\{A, R A\}$. The second statement already appeared in the previous section (see (c), first paragraph). Self-duality transforms the second statement into the first by Lemma 1. In addition, we provide the next Lorenz-based characterization of the Talmud rule.

Proposition 6 Restrict the domain to $\mathcal{C}_{0}$. Let $\mathcal{R}$ be the set of rules that satisfy order preservation of awards, order preservation of losses, resource monotonicity, and the midpoint property. The Talmud rule $\left(\left.T\right|_{\mathcal{C}_{0}}\right)$ is the only rule in $\mathcal{R}$ that is Lorenz dominated by each rule in $\mathcal{R}$ on the domain $\mathcal{C}_{0} .{ }^{12}$

Proof Resource monotonicity and the midpoint property are self-dual properties: if a rule $R$ satisfies these properties, then so does $R^{d}$ (Thomson 2003). Also, the domains $\mathcal{C}_{0}$ and $\mathcal{C}_{1}$ are dual. Proposition 2 implies that, on the domain $\mathcal{C}_{1}, T$ Lorenz dominates each rule that satisfies order preservation of awards, order preservation of losses, resource monotonicity, and the midpoint property. Take the dual of this implication and apply Lemma 1.

Table 4 presents an overview of the propositions.

We conclude by illustrating the incomparabilities. In each case, we present an example in the domain $\mathcal{C}_{1}$. If the incomparability also holds in the domain $\mathcal{C}_{0}$, then the duals provide an illustration. Each example involves a small number of individuals. To increase this number, just add individuals with zero claims and use limited consistency.

(d) $R A$ and $P$ are incomparable in $\mathcal{C}_{0}$ and in $\mathcal{C}_{1}$. The problems in Tables 1 and 5 illustrate that the random arrival rule and the proportional rule are incomparable in $\mathcal{C}_{1}$.

\footnotetext{
12 See Hougaard and Thorlund-Petersen (2001, Theorem 2) for a related result.
} 
Table 4 A summary of the Lorenz-based characterizations

\begin{tabular}{|c|c|c|c|c|c|c|}
\hline & \multicolumn{6}{|c|}{ Proposition } \\
\hline & 1 & 2 & 3 & 4 & 5 & 6 \\
\hline Lorenz-maximal & $C E A$ & $C E\left(T \mid \mathcal{C}_{1}\right)$ & Pin & & & \\
\hline Order preservation of awards & $\bullet$ & $\bullet$ & $\bullet$ & - & $\bullet$ & $\bullet$ \\
\hline Midpoint property & & $\bullet$ & $\bullet$ & & & $\bullet$ \\
\hline Resource monotonicity & & $\bullet$ & $\bullet$ & & & $\bullet$ \\
\hline Super-modularity & & & $\bullet$ & & & \\
\hline Order preservation under claims & & & & & & \\
\hline variations & & & & • & & \\
\hline Reasonable lower bounds on awards & & & & $\bullet$ & & \\
\hline Order preservation of losses & & & & $\bullet$ & $\bullet$ & $\bullet$ \\
\hline Lorenz-minimal & & & & $M O$ & $C E L$ & $\left.T\right|_{\mathcal{C}_{0}}$ \\
\hline
\end{tabular}

Table 5 Example with $c=(1000,2500,2500)$ and $E=2700$

\begin{tabular}{lrrr}
\hline Claims & $P$ & $R A$ & $M O$ \\
\hline 1000 & 450 & 400 & 333 \\
2500 & 1125 & 1150 & 1183 \\
2500 & 1125 & 1150 & 1183 \\
\hline 6000 & 2700 & 2700 & 2700 \\
\hline
\end{tabular}

(e) $R A$ and $A$ are incomparable in $\mathcal{C}_{0}$ and in $\mathcal{C}_{1}$. The problem in Table 1 and the problem $(c, E)=(10,10,10,20,20)$ show that the random arrival rule and the adjusted proportional rule are incomparable in $\mathcal{C}_{1}$. Indeed, $R A(c, E)=(4.2,4.2,4.2,7.5)$ Lorenz dominates $A(c, E)=(4,4,4,8)$.

(f) $M O$ and $P$ are incomparable in $\mathcal{C}_{1}$. The problems in Tables 1 and 5 show that the minimal overlap rule and the proportional rule are incomparable in $\mathcal{C}_{1}$.

This completes the proof of Theorem 2 .

Acknowledgments We thank Bart Capéau, Frank Cowell, Juan Moreno-Ternero, Erik Schokkaert, Frans Spinnewyn, William Thomson, and Bertil Tungodden for their comments. We also thank an anonymous associate editor and two anonymous referees for their helpful suggestions. All remaining shortcoming are ours. Kristof Bosmans gratefully acknowledges financial support from the Fund for Scientific ResearchFlanders (grant G.0005.04) and the Interuniversity Attraction Poles network funded by the Federal Public Planning Service, Belgian Science Policy (grant P5/21-A).

Open Access This article is distributed under the terms of the Creative Commons Attribution Noncommercial License which permits any noncommercial use, distribution, and reproduction in any medium, provided the original author(s) and source are credited. 


\section{References}

Bosmans K, Schokkaert E (2009) Equality preference in the claims problem: a questionnaire study of cuts in earnings and pensions. Soc Choice Welf 33:533-557

Chun Y, Thomson W (2005) Convergence under replication of rules to adjudicate conflicting claims. Games Econ Behav 50:129-142

Chun Y, Schummer J, Thomson W (2001) Constrained egalitarianism: a new solution for claims problems. Seoul J Econ 14:269-297

Dominguez D, Thomson W (2006) A new solution to the problem of adjudicating conflicting claims. Econ Theory 28:283-307

Herrero C, Villar A (2001) The three musketeers: four classical solutions to bankruptcy problems. Math Soc Sci 42:307-328

Hougaard JL, Thorlund-Petersen L (2001) Bankruptcy rules, inequality, and uncertainty. Working Paper 4/01, Department of Operations Management, Copenhagen Business School

Moreno-Ternero JD, Villar A (2004) The Talmud rule and the securement of agents' awards. Math Soc Sci 47:245-257

Moreno-Ternero JD, Villar A (2006) On the relative equitability of a family of taxation rules. J Public Econ Theory 8:283-291

Moulin H (2002) Axiomatic cost and surplus sharing. In: Arrow KJ, Sen AK, Suzumura K (eds) Handbook of social choice and welfare. Elsevier, Amsterdam, pp 289-357

Schummer J, Thomson W (1997) Two derivations of the uniform rule and an application to bankruptcy. Econ Lett 55:333-337

Sen AK (1973) On economic inequality. Clarendon Press, Oxford

Thomson W (2002) Two families of rules for the adjudication of conflicting claims. Mimeo

Thomson W (2003) Axiomatic and game-theoretic analysis of bankruptcy and taxation problems: a survey. Math Soc Sci 45:249-297

Thomson W (2006) How to divide when there is not enough; from the Talmud to game theory. Unpublished book manuscript

Thomson W (2011) Lorenz rankings of rules for the adjudication of conflicting claims. Econ Theory. doi:10.1007/s00199-010-0575-5

Thomson W, Yeh C-H (2006) Operators for the adjudication of conflicting claims. Rochester Center for Economic Research Working Paper 531, University of Rochester 